\title{
A Full Discrete Dispersion Analysis of Time-Domain Simulations of Acoustic Liners with Flow
}

\author{
G. Gabard ${ }^{\mathrm{a}}$, E.J. Brambley ${ }^{\mathrm{b}}$ \\ ${ }^{a}$ Institute of Sound and Vibration Research, University of Southampton \\ University Road, Southampton, SO17 1BJ, United Kingdom \\ ${ }^{b}$ Department of Applied Mathematics and Theoretical Physics, University of Cambridge \\ Wilberforce Road, Cambridge, CB3 OWA, United Kingdom
}

\begin{abstract}
The effect of flow over an acoustic liner is generally described by the Myers impedance condition. The use of this impedance condition in time-domain numerical simulations has been plagued by stability issues, and various ad hoc techniques based on artificial damping or filtering have been used to stabilise the solution. The theoretical issue leading to the ill-posedness of this impedance condition in the time domain is now well understood. For computational models, some trends have been identified, but no detailed investigation of the cause of the instabilities in numerical simulations has been undertaken to date. This paper presents a dispersion analysis of the complete numerical model, based on finite-difference approximations, for a twodimensional model of a uniform flow above an impedance surface. It provides insight into the properties of the instability in the numerical model and clarifies the parameters that influence its presence. The dispersion analysis is also used to give useful information about the accuracy of the acoustic solution.

Comparison between the dispersion analysis and numerical simulations shows that the instability associated with the Myers condition can be identified in the numerical model, but its properties differ significantly from that of the continuous model. The unbounded growth of the instability in the continuous model is not present in the numerical model due to the wavenumber aliasing inherent to numerical approximations. Instead, the numerical instability includes a wavenumber component behaving as an absolute instability. The trend previously reported that the instability is more likely to appear with fine grids is explained. While the instability in the numerical model is heavily dependent on the spatial resolution, it is well resolved in time and is not sensitive to the time step. In addition filtering techniques to stabilise the solutions are considered and it is found that, while they can reduce the instability in some cases, they do not represent a systematic or robust solution in general.
\end{abstract}




\section{Introduction}

Acoustic linings and the effects of their presence, position, and properties continue to be of major importance to sound reduction in aeroengines. The boundary condition often used to model acoustics in flow over acoustic linings is the Myers [27, or Ingard-Myers [20, boundary condition. This boundary condition, formulated in the frequency-domain, relates the acoustic pressure $\hat{p} \mathrm{e}^{\mathrm{i} \omega t}$ and acoustic normal velocity $\hat{v}_{n} \mathrm{e}^{\mathrm{i} \omega t}$ at the lining in order to give a specified lining impedance $\hat{Z}(\omega)$. The Myers boundary condition is given by

$$
\mathrm{i} \omega \hat{v}_{n}=\left[\mathrm{i} \omega+\mathbf{u}_{0} \cdot \boldsymbol{\nabla}-\left(\mathbf{n} \cdot \boldsymbol{\nabla} \mathbf{u}_{0}\right) \cdot \mathbf{n}\right] \frac{\hat{p}}{\hat{Z}},
$$

where $\mathbf{u}_{0}$ is the mean flow velocity and $\mathbf{n}$ is the lining surface normal. This boundary condition correctly represents the limit of a vanishingly-thin inviscid boundary layer over the acoustic lining [16, 39], although the boundary layer needs to be extremely thin in some cases for this to be a good approximation [15]. A brief review of the mathematical modelling of acoustic linings with flow is given in Ref. 6 .

Since the Myers boundary condition is naturally expressed in the frequency-domain, a number of techniques have been proposed for its adaption for use in time-domain numerical simulations for various different impedance models. Özyörük et al. [28] implemented the Myers boundary condition in a time-domain finite difference simulation, modelling the lining impedance $\hat{Z}(\omega)$ by a rational function of $\omega$ and applying a $z$-transform. Both Ju \& Fung [21] and Li et al. 23] performed time-domain finite difference simulations modelling the acoustic lining using a modification of the three parameter model of Tam and Auriault [36] with the Myers boundary condition incorporated into an effective impedance; 23] did so by modifying the impedance $\hat{Z}$, while Ju \& Fung [21] modified the reflection coefficient $\hat{W}=(1-\hat{Z}) /(1+\hat{Z})$. The three parameter acoustic lining model was subsequently implemented directly using the Myers boundary condition in a time-domain finite difference simulation by Tam et al. [37. Chevaugeon et al. [12] implemented the Myers boundary condition in a time-domain discontinuous Galerkin simulation, modelling the acoustic lining as an Extended Helmholtz Resonator [33. The Myers boundary condition with the Extended Helmholtz Resonator model may also be implemented in finite-difference simulations using a $z$-transform, as suggested by Rienstra [33, and demonstrated by Richter et al. [31.

A number of theoretical [32, 10, 5, 34, 24, 7, 25) and experimental [11, 1, 26, studies suggest that, in certain circumstances, flow over an acoustic lining should give rise to an instability. This physical instability could be one reason for the instability seen in time-domain simulations using the Myers boundary condition. However, Brambley [4] suggested the root cause of the numerical instability of the Myers boundary condition is that the underlying mathematical problem is ill-posed. This was demonstrated using a dispersion analysis, which considers waves of the form $\mathrm{e}^{\mathrm{i} \omega t-\mathrm{i} k x}$ where the frequency $\omega$ and wavenumber $k$ are linked by a dispersion relation $D(k, \omega)=0$. This dispersion relation may be solved to give $\omega$ as a function of $k$, which is equivalent to solving the initial value problem for the time evolution of initial conditions $\mathrm{e}^{-\mathrm{i} k x}$. For bounded initial conditions, implying $k$ is real, the stability of the subsequent evolution may be characterised by the exponential growth rate $-\operatorname{Im}(\omega(k))$. For the Myers boundary condition, Brambley [4] showed this growth rate to be unbounded as $k \rightarrow \infty$, implying that the problem is ill-posed. This is also the reason why a rigorous stability analysis is not possible for the Myers boundary condition, leading to the continuing debate over stability. However, to date, there has been no conclusive evidence directly linking the ill-posedness of the mathematical problem highlighted by Brambley [4] to the observed numerical instabilities in time-domain simulations.

In each of the computational studies mentioned above, filtering (or equivalently artificial dissipation) was found necessary to prevent high-frequency under-resolved oscillations and ensure stability. While it is known that filtering to remove high-frequency under-resolved oscillations is necessary with low-dispersion low-dissipation time-domain schemes [2], in practice the filtering needed to stabilise the Myers boundary condition is far greater than that needed to ensure stability within the bulk of the fluid; for example, Richter 29] found it necessary to use a very strong 3-point filter on part of the normal velocity $v_{n}$ prescribed by the Myers boundary condition. In general, the strength of filtering has to be adjusted on a case-by-case basis, since the strength of the instability varies with the grid resolution, the numerical scheme, the liner impedance $\hat{Z}$ and the flow velocity $\mathbf{u}_{0}$. In practice, therefore, it is difficult to ensure a stable simulation, 
and also difficult to assess the impact of the additional filtering on the prediction of sound attenuation by the lining. Moreover, use of this filtering implicitly assumes that flow over acoustic linings is stable, which may not reflect reality [11, 1, 26].

This paper investigates the numerical instability of time-domain simulations using the Myers boundary condition by performing a dispersion analysis of the discretised problem. The idea of a dispersion analysis of a discretization scheme is not new and a general description of this type of analyses can be found in 41 . Trefethen [40] gave a discussion of the dispersion relation for several finite-difference schemes applied to a 1D advection equation and a 2D wave equation, including the calculation and interpretation of the numerical group velocity. A similar concept was used by Tam \& Webb [38] in creating Dispersion Relation Preserving (DRP) finite-difference schemes, where the finite-difference stencils were designed so that the numerical dispersion relation was a good approximation to the continuous dispersion relation of the continuous system being solved. More recently, Cossu \& Loiseleux [13] considered the dispersion relation of three finitedifference schemes for solving the linearised Ginzburg-Landau equation, and interpreted their results in terms of convective and absolute instability by considering the numerical group velocity. However, each of these studies performed a dispersion analysis of only the spatial and temporal finite-difference schemes. In contrast, in this paper we perform a dispersion analysis of the entire numerical method, including not only the spatial and temporal finite-difference schemes but also the numerical implementation of the Myers boundary condition, the artificial filtering, and the non-reflecting boundary conditions at the edges of the computational domain. To our knowledge, this is the first time such a complete discretised dispersion analysis has been performed. This numerical dispersion analysis is used: (i) to compare the instability observed in time-domain simulations to the unstable mode predicted by the continuous model; (ii) to provide insight into the discrepancies between the finite-difference approximation and the continuous model; and (iii) to study the parameters of the numerical model that can influence the strength of the instability.

This paper is organised as follows. The benchmark problem and the numerical methods are described in the next section, and the presence of unstable solutions is illustrated. In section 3 the dispersion analysis of the numerical model is introduced. Then in section 4 the dispersion analysis is used and compared with the time-domain simulation to better understand the properties of the instabilities associated with the liner. Finally, in section 5 , it is shown how this dispersion analysis can provide useful information on other aspects of the numerical model.

\section{Numerical model}

\subsection{Problem description}

To discuss the properties of numerical models based on the Myers impedance condition, we consider a two-dimensional problem, shown in figure 2.1, with a uniform mean flow in the $x$ direction with velocity $u_{0}$, sound speed $c_{0}$ and density $\rho_{0}$. The propagation of linear disturbances on this mean flow is described by the linearised Euler equations (LEE) which may be written as

$$
\frac{\partial \mathbf{q}}{\partial t}+\mathbf{F}_{x} \frac{\partial \mathbf{q}}{\partial x}+\mathbf{F}_{y} \frac{\partial \mathbf{q}}{\partial y}=\mathbf{s}
$$

with

$$
\mathbf{q}=\left[\begin{array}{c}
\rho^{\prime} \\
(\rho u)^{\prime} \\
(\rho v)^{\prime} \\
p^{\prime}
\end{array}\right], \mathbf{F}_{x}=\left[\begin{array}{cccc}
0 & 1 & 0 & 0 \\
-u_{0}^{2} & 2 u_{0} & 0 & 1 \\
0 & 0 & u_{0} & 0 \\
-u_{0} c_{0}^{2} & c_{0}^{2} & 0 & u_{0}
\end{array}\right], \mathbf{F}_{y}=\left[\begin{array}{cccc}
0 & 0 & 1 & 0 \\
0 & 0 & u_{0} & 0 \\
0 & 0 & 0 & 1 \\
0 & 0 & c_{0}^{2} & 0
\end{array}\right]
$$

where $u^{\prime}$ and $v^{\prime}$ are the acoustic velocity components, and $\rho^{\prime}$ and $p^{\prime}$ are the acoustic density and pressure.

Starting from the initial condition $\mathbf{q}=\mathbf{0}$ at $t=0$, the solution is driven by the source $\mathbf{s}$ which is a time-harmonic acoustic monopole corresponding to a mass source located at $\left(x_{s}, y_{s}\right)$ and defined by

$$
\mathbf{s}=\left[\begin{array}{c}
1 \\
u_{0} \\
0 \\
c_{0}^{2}
\end{array}\right] \exp \left[-\frac{\left(x-x_{s}\right)^{2}+\left(y-y_{s}\right)^{2}}{2 w^{2}}\right] \frac{\sin (\omega t)}{2 \pi w^{2}},
$$




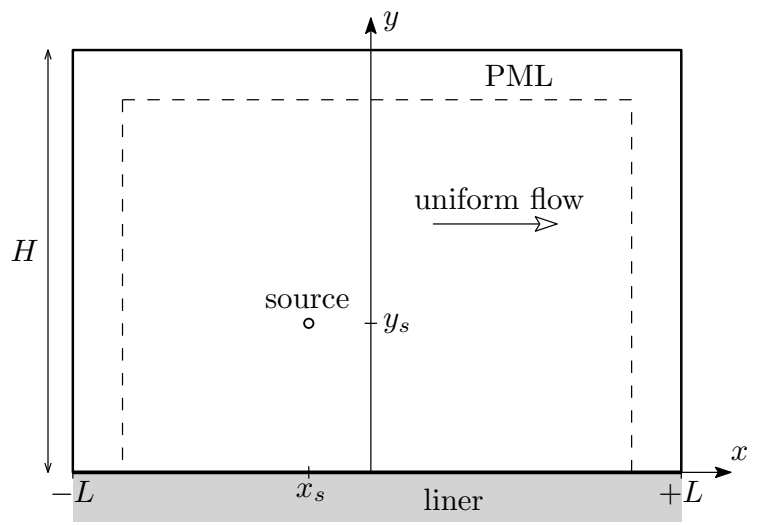

Figure 2.1: Diagram of the computational test case.

where $w$ is the width of the source and $\omega$ its angular frequency.

The acoustic waves radiating from the source interact with an acoustically lined surface located at $y=0$. This surface is characterised by its acoustic impedance $\hat{Z}(\omega)$ given in the frequency domain (with an $\mathrm{e}^{+\mathrm{i} \omega t}$ notation). The interaction between the liner and the sound field will be described using the Myers condition 27]:

$$
\mathrm{i} \omega \hat{v}_{n}(x, \omega)=\left(\mathrm{i} \omega+u_{0} \frac{\partial}{\partial x}\right)\left[\frac{\hat{p}(x, 0, \omega)}{\hat{Z}(\omega)}\right]
$$

where^denotes frequency-domain quantities, and $\hat{v}_{n}=-\hat{v}(x, y=0, \omega)$ is the normal velocity. This condition assumes an infinitely thin boundary layer, and effectively uses a vortex sheet model where the continuity of pressure and normal displacement is applied [16, 39, 15.

In the limit of an infinitely small source $(w \rightarrow 0)$ the present test case is equivalent to the benchmark problem proposed in reference 8 , and for which a complete analytical solution has been made available.

\subsection{Finite-difference approximations}

The field equations 2.1) are solved in the time domain using finite-difference schemes on a rectangular computational domain defined by $-L<x<L$ and $0<y<H$. The node spacing on the Cartesian grid is denoted by $\Delta x$ and $\Delta y$. Spatial derivatives are approximated using a 7 -point, dispersion-relation-preserving scheme optimised for low dispersion [38, 2]. For time marching a Runge-Kutta scheme optimised for low dispersion and low dissipation is used with time step $\Delta t$ [3]. A selective filter is applied at each time step to remove the spurious, short-wavelength components that are supported by low-dissipation schemes [3]. These are widely used schemes and the analysis presented in this paper can also be performed with other numerical methods such as compact schemes for instance. The results of the analysis performed here are expected to be typical of any such finite difference scheme.

\subsection{Impedance condition}

Various formulations of impedance have been considered for time-domain simulations of acoustic liners. Here we will use the simple and well-known model of a mass-spring-damper resonator to describe the response of the liner to the sound field. This corresponds to an impedance given by

$$
\hat{Z}(\omega)=R+\mathrm{i} M \omega-\mathrm{i} K / \omega,
$$

where $M, K$ and $R$ are the mass, spring stiffness and damping coefficients (per unit length) of the resonator.

Other popular impedance models include the Extended Helmholtz Resonator proposed by Rienstra [33, the three-parameter model by Tam and Auriault [36, and broadband impedance models [28. They differ by the way they are formulated and implemented in the time domain, as well as their ability to represent 
realistic impedance functions. It should be emphasised that the interest of this paper is in investigating the behaviour of the boundary condition (2.2), and not in the specific impedance model (2.3) used. It is anticipated that what follows will be similarly applicable to other models of the impedance.

\subsubsection{Time-domain formulation}

The key for time-domain simulations of impedance models is the calculation of the quantity:

$$
\hat{\eta}(x, \omega)=\hat{p}(x, 0, \omega) / \hat{Z}(\omega) .
$$

which, when written in the time domain, represents a convolution product between the acoustic pressure on the liner and an impulse response function for the liner (the corresponding frequency response function is in fact the admittance $\hat{A}(\omega)=1 / \hat{Z}(\omega))$. This must be done in such a way that a number of basic physical principles such as causality are satisfied 33 .

The implementation of the impedance depends strongly on the type of impedance function $\hat{Z}(\omega)$ that is assumed. The mass-spring-damper model defined by $(2.3)$ can be formulated in the time domain with the following coupled equations:

$$
\begin{aligned}
& \frac{\partial \eta(x, t)}{\partial t}=\frac{1}{M}\left[p^{\prime}(x, 0, t)-K \xi(x, t)-R \eta(x, t)\right], \\
& \frac{\partial \xi(x, t)}{\partial t}=\eta(x, t) .
\end{aligned}
$$

This implementation of the impedance condition satisfies all the conditions given in 33 .

The second auxiliary variable $\xi$ corresponds to the displacement of the mass-spring-damper (this is also the displacement of the fluid). The variable $\eta$ corresponds to the normal velocity between the vortex sheet and the liner, in contrast to the normal velocity $v_{n}$ which is defined above the vortex sheet. The Myers condition 2.2, written in the time domain, relates the two normal velocities $\eta$ and $v_{n}$ on either sides of the vortex sheet:

$$
\frac{\partial v_{n}(x, t)}{\partial t}=\frac{\partial \eta(x, t)}{\partial t}+u_{0} \frac{\partial \eta(x, t)}{\partial x} .
$$

The time derivative of $\eta$ is directly provided by 2.4 and we have

$$
\frac{\partial v_{n}}{\partial t}=\frac{1}{M} p^{\prime}-\frac{K}{M} \xi-\left(\frac{R}{M}+u_{0} \frac{\partial}{\partial x}\right) \eta .
$$

The second term on the right-hand side of 2.6 is often called the convective term, and the spatial derivative is calculated using the same finite-difference stencils as for the LEE.

Equations (2.4) and (2.5) are easily implemented by defining the two auxiliary variables $\eta$ and $\xi$ at each grid point on the impedance boundary. These variables are integrated in time using (2.4) and (2.5) and the local value of pressure. The same time-marching scheme as for the LEE is used. Note that equations (2.4) and 2.5) are purely local since they do not require information from neighbouring grid points.

\subsubsection{Implementation}

Equation (2.6) is used to modify the field equations $(2.1)$ solved along the impedance surface $y=0$. A straightforward approach is to substitute the third equation in (2.1) by

$$
\frac{\partial(\rho v)^{\prime}}{\partial t}=-\rho_{0}\left[\frac{1}{M} p^{\prime}-\frac{K}{M} \xi-\left(\frac{R}{M}+u_{0} \frac{\partial}{\partial x}\right) \eta\right], \quad \text { at } y=0 .
$$

We will show however in section 5.2 that this approach can lead to numerical instabilities.

Another approach is to use the characteristic waves of the governing equations (2.1) to modify them in a way that satisfies the boundary condition 2.6) (see reference [18] for a detailed discussion of the link between characteristics and well-posed boundary conditions). This approach is presented here for the case 
of linear equations with constant coefficients (for non-linear problems see reference [22]). To define the onedimensional characteristic waves along the normal to the lined surface, we introduce the normal flux matrix $\mathbf{F}_{n}=-\mathbf{F}_{y}$. We then calculate the matrix $\mathbf{W}$ of eigenvectors of $\mathbf{F}_{n}$ and the diagonal matrix $\boldsymbol{\Lambda}$ of eigenvalues, which correspond to the phase speeds of the characteristic waves. The amplitudes of the characteristic waves can then be defined as $\tilde{\mathbf{q}}=\mathbf{W}^{-1} \mathbf{q}$. For the linearised Euler equations 2.1 , we have

$$
\tilde{\mathbf{q}}=\left[\begin{array}{c}
\tilde{q}_{v} \\
\tilde{q}_{e} \\
\tilde{q}_{a}^{+} \\
\tilde{q}_{a}^{-}
\end{array}\right], \mathbf{W}=\left[\begin{array}{cccc}
0 & 1 & 1 & 1 \\
1 & u_{0} & u_{0} & u_{0} \\
0 & 0 & -c_{0} & c_{0} \\
0 & 0 & c_{0}^{2} & c_{0}^{2}
\end{array}\right], \boldsymbol{\Lambda}=\operatorname{diag}\left(0,0,+c_{0},-c_{0}\right)
$$

where we have used the fact that the mean flow is tangential to the boundary $\left(\mathbf{u}_{0} \cdot \mathbf{n}=0\right)$. The quantities $\tilde{q}_{e}$ and $\tilde{q}_{v}$ represent the amplitudes of the entropy and vorticity waves, while $\tilde{q}_{a}^{+}$and $\tilde{q}_{a}^{-}$are the amplitudes of the acoustic characteristics propagating into and out of the lining, respectively.

As explained by Higdon [18, the boundary condition must be such that the incoming characteristics are completely specified. For an impedance surface, there is only one incoming characteristic wave $\tilde{q}_{a}^{-}$. From the definition $\mathbf{q}=\mathbf{W}^{-1} \tilde{\mathbf{q}}$ we can write:

$$
\frac{\partial \tilde{q}_{a}^{-}}{\partial t}=\frac{\partial \tilde{q}_{a}^{+}}{\partial t}-\frac{\rho_{0}}{c_{0}} \frac{\partial v_{n}}{\partial t},
$$

which is used to write the amplitude $\tilde{q}_{a}^{-}$of the acoustic wave entering the domain in terms of $v_{n}$ and $\tilde{q}_{a}^{+}$. This expression is then recast in terms of the physical variables $\mathbf{q}$ and used to modify the governing equations along the liner as follows:

$$
\frac{\partial \mathbf{q}}{\partial t}=\mathbf{W R W}^{-1}\left(\mathbf{s}-\mathbf{F}_{x} \frac{\partial \mathbf{q}}{\partial x}-\mathbf{F}_{y} \frac{\partial \mathbf{q}}{\partial y}\right)-\frac{\rho_{0}}{c_{0}} \frac{\partial v_{n}}{\partial t} \mathbf{w}_{a}^{-}, \quad \text { for } y=0
$$

where $\partial v_{n} / \partial t$ is given by 2.6$), \mathbf{w}_{a}^{-}$is the column of $\mathbf{W}$ corresponding to $\tilde{q}_{a}^{-}$, and $\mathbf{R}$ is a reflection matrix for the characteristics:

$$
\mathbf{w}_{a}^{-}=\left[\begin{array}{c}
1 \\
u_{0} \\
c_{0} \\
c_{0}^{2}
\end{array}\right], \quad \mathbf{R}=\left[\begin{array}{cccc}
1 & 0 & 0 & 0 \\
0 & 1 & 0 & 0 \\
0 & 0 & 1 & 0 \\
0 & 0 & 1 & 0
\end{array}\right] .
$$

\subsection{Perfectly matched layers}

To simulate an infinite domain, Perfectly Matched Layers (PML) are implemented at the upper and lateral boundaries. The stable PML formulation proposed by $\mathrm{Hu} 19$ for a uniform flow is used here. The modified field equations in the PML regions are

$$
\frac{\partial \mathbf{q}}{\partial t}+\mathbf{F}_{x} \frac{\partial \mathbf{q}}{\partial x}+\mathbf{F}_{y} \frac{\partial \mathbf{q}}{\partial y}+\sigma_{x} \mathbf{F}_{y} \frac{\partial \mathbf{h}}{\partial y}+\sigma_{y} \mathbf{F}_{x} \frac{\partial \mathbf{h}}{\partial x}+\left(\sigma_{x}+\sigma_{y}\right) \mathbf{q}+\sigma_{x} \sigma_{y} \mathbf{h}+\frac{\sigma_{x} M}{1-M^{2}} \mathbf{F}_{x}\left(\mathbf{q}+\sigma_{y} \mathbf{h}\right)=\mathbf{0}
$$

where $M=u_{0} / c_{0}$ is the mean flow Mach number. We have introduced the auxiliary variable $\mathbf{h}$ such that

$$
\frac{\partial \mathbf{h}}{\partial t}=\mathbf{q}
$$

The absorption coefficients $\sigma_{x}$ and $\sigma_{y}$ vary from 0 inside the physical region of the computational domain to $\sigma_{\max }$ at the boundary (a quadratic function is used for this purpose). The strength of the PML is defined by $\sigma_{\max }=1 / \Delta x$. 


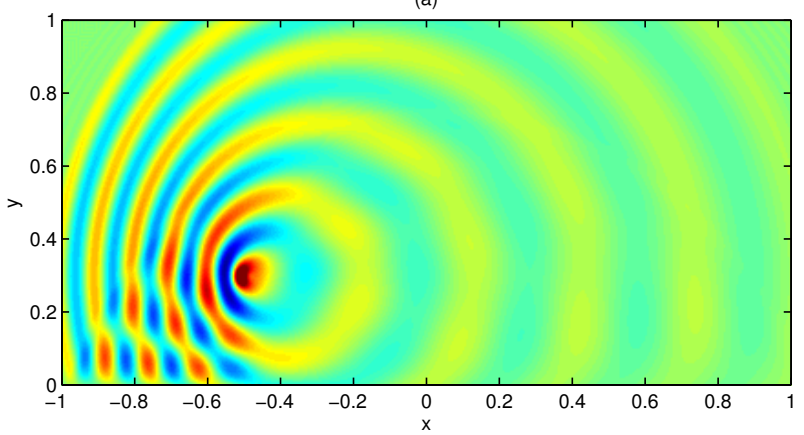

(c)

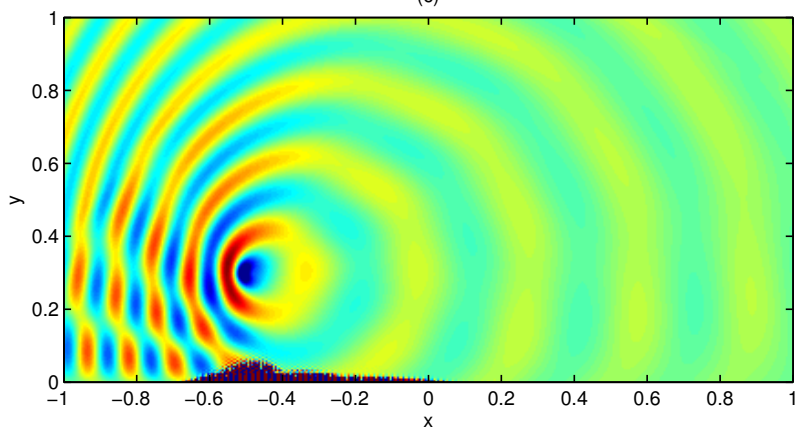

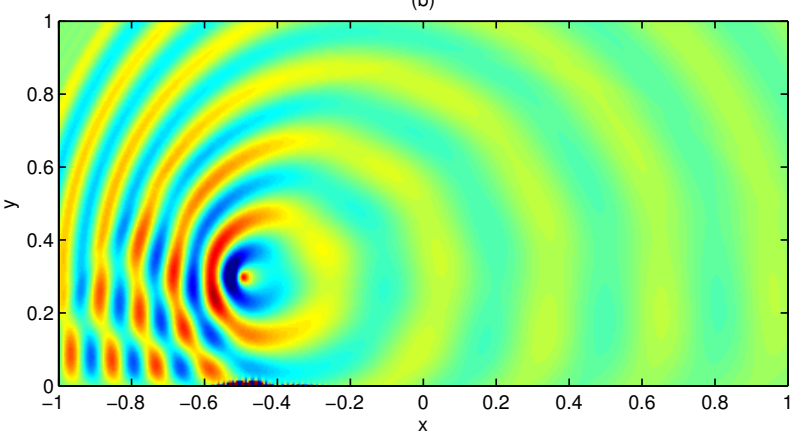

(d)

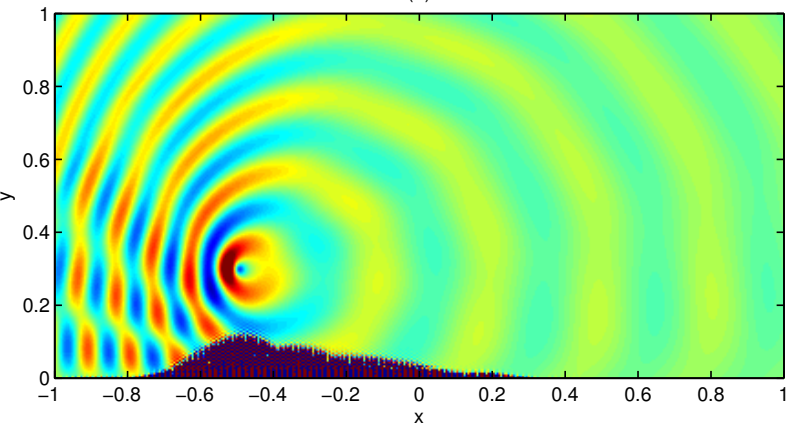

Figure 2.2: Snapshots of pressure in the numerical simulation at (a) $t=350 \Delta t$, (b) $t=400 \Delta t$, (c) $t=450 \Delta t$ and (d) $t=500 \Delta t$. Note the rapid growth of an instability at the impedance surface $y=0$.

\subsection{Example of results}

To illustrate the results obtained with this numerical model, we consider a test case where the mean flow properties are $\rho_{0}=1, c_{0}=1$ and $u_{0}=0.5$. The source is located at $x_{s}=-0.5, y_{s}=0.3$ with angular frequency $\omega=31$ and width $w=0.008$. The property of the mass-spring-damper model for the liner are $M=0.01, K=10$ and $R=0.75$. The size of the computational domain is $L=2.5$ and $H=2$. The time step corresponds to a CFL number $\left(u_{0}+c_{0}\right) \Delta t / \Delta x=0.9$. Unless stated otherwise a grid resolution $\Delta x=\Delta y=0.005$ is used, corresponding to at least 20 points per wavelength for the acoustic waves, and a time step $\Delta t=0.003$. The width of the PMLs is 15 grid points. In some cases a coarse grid with $\Delta x=\Delta y=0.01$ will be used.

Figure 2.2 shows the evolution of the pressure field at several time steps. Apart from the reflection of the incident sound field by the impedance surface, the key feature to note is the growth of an instability from the boundary. This instability dominates the acoustic waves by several orders of magnitude and rapidly propagates through the computational domain.

A complete analytical solution for this benchmark problem is provided in reference [8] and it includes a surface wave which can be treated as an instability. The analytical solution is shown in figure 2.3 , and this unstable surface wave can be seen to emanate from a point on the liner located downstream of the source. In $2.3 \mathrm{~b}$ the numerical solution is compared against the reference solution, with the unstable wave subtracted for clarity. The excellent agreement observed indicates that the numerical model represents accurately the propagation and absorption of sound. This also indicates that the instability visible in the theoretical solution is not present in the numerical model and instead we observe a very different form of instability with a very small wavelength as seen in figure 2.2 

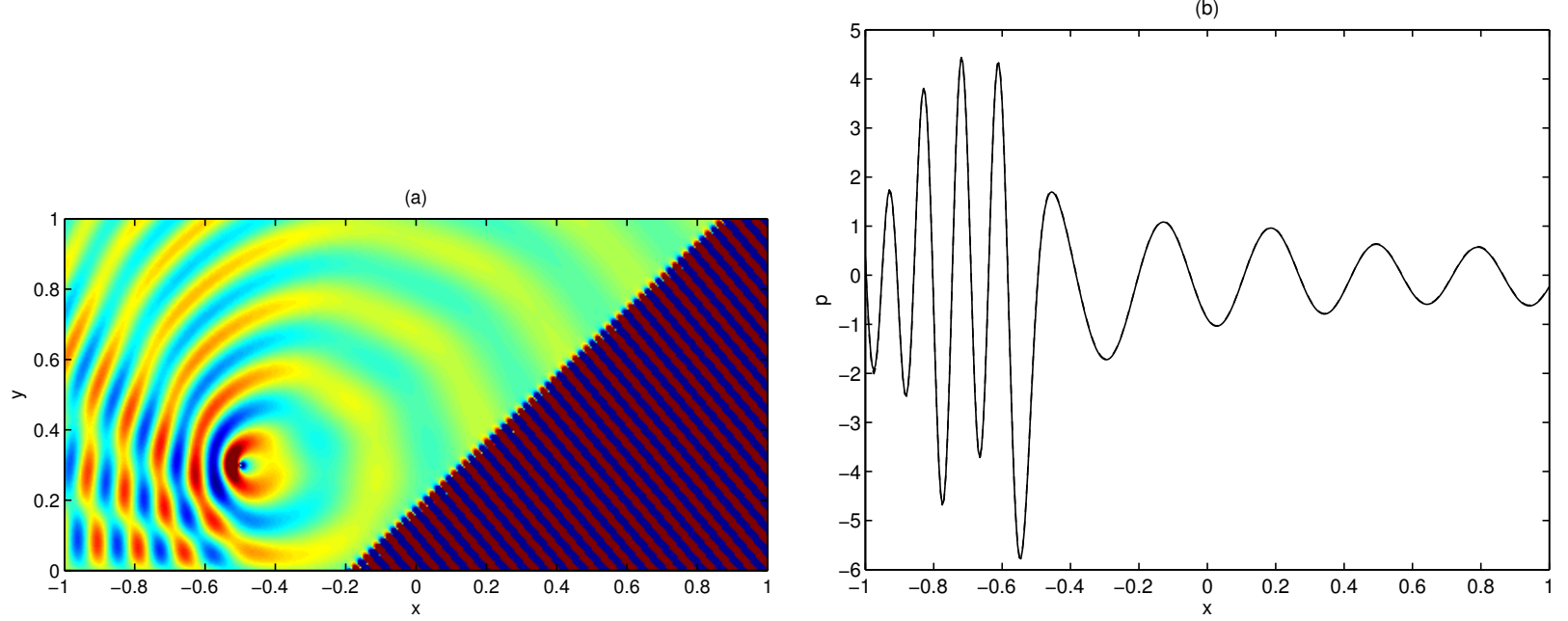

Figure 2.3: (a) Analytical solution with the unstable mode. (b) Comparison of the analytical solution without the unstable mode (dashed line) at $y=0.4$ with the numerical solution (solid line) at $t=500 \Delta t$ shown in figure $2.2 \mathrm{~d}$.

\section{Dispersion Analysis}

We now present a dispersion analysis of the numerical model, so as to better understand the occurrence of instabilities in the numerical simulation and the factors contributing to their presence. For the purpose of this analysis the computational domain is considered infinite in the $x$ direction and we assume numerical solutions of the form $\mathbf{q} \sim \mathrm{e}^{-\mathrm{i} k x}$, which is similar to the dispersion analysis of the continuous model presented in references [4, 5]. For the numerical model, this leads to a fully discrete eigenvalue problem, and we can study the dispersion properties of the numerical solution in terms of the angular frequency $\omega(k)$ of each mode.

We consider a uniform grid with spacing $\Delta x$ and $\Delta y$, as shown schematically in figure 3.1. The coordinates of the grid points are given by $x_{m}=m \Delta x$ and $y_{n}=n \Delta y$. We denote the solution at each grid point by $\mathbf{q}_{m, n}(t)$. With the assumed wave-like behaviour $\mathrm{e}^{-\mathrm{i} k x}$, the solution can be written

$$
\mathbf{q}_{m, n}(t)=\mathbf{q}_{0, n}(t) \mathrm{e}^{-\mathrm{i} k m \Delta x},
$$

and we are left with a finite number of degrees of freedom $\mathbf{q}_{0, n}$. Since we are solving a linear system of equations, it is possible to write the solution at a given time step as a linear combination of the solution at the previous time step:

$$
\mathbf{p}(t+\Delta t)=\mathbf{K p}(t),
$$

where the vector $\mathbf{p}$ contains all the degrees of freedom, that is $\mathbf{q}_{0, n}(t)$ together with the auxiliary variables $\eta$ and $\xi$ introduced for the Myers impedance condition, and the variables $\mathbf{h}$ introduced for the PML. As shown in detail in sections 3.1 to 3.4, the coefficient matrix $\mathbf{K}$ contains all the operations performed when marching the solution in time from $t$ to $t+\Delta t$, including the finite-difference approximation of the flux divergence, the implementation of the impedance condition, the upper buffer zone, the time integration and the selective filter applied at each time step.

The growth rate of the solution between two time steps can be defined by the coefficient $\lambda$ such that $\mathbf{p}(t+\Delta t)=\lambda \mathbf{p}(t)$. This leads to the following eigenvalue problem $\mathbf{K z}=\lambda \mathbf{z}$. Alternatively we can consider the angular frequency $\omega$ of the modes such that $\lambda=\mathrm{e}^{+\mathrm{i} \omega \Delta t}$. We obtain a set of modes, each with an angular frequency $\omega(k)$ and eigenvector $\mathbf{z}(k)$ parametrised by the wavenumber $k$. This provides a description of the dispersion properties of the numerical model. For each mode we can define the phase speed $\omega / k$ and group velocity $\mathrm{d} \omega / \mathrm{d} k$ along the $x$ axis. In particular we are interested in the presence of unstable modes for 


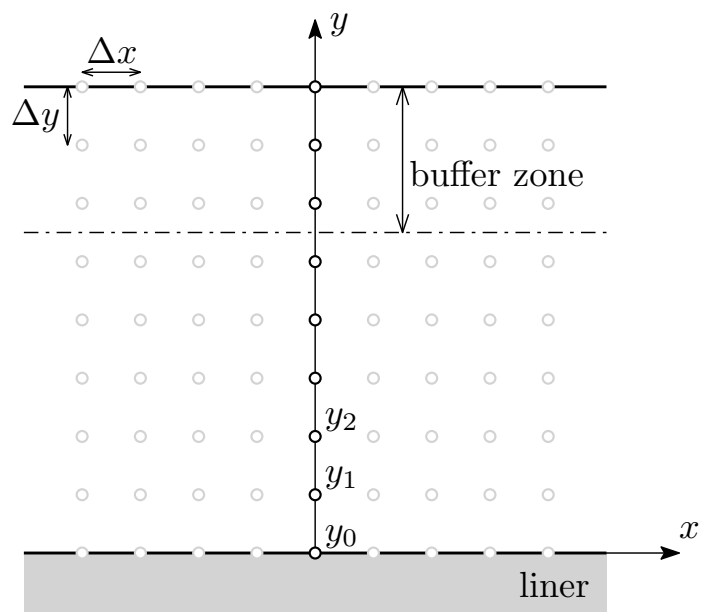

Figure 3.1: Diagram of the problem for the stability analysis.

which $\operatorname{Im}(\omega)<0$ (or equivalently $|\lambda|>1$ ). The discretised system (3.1) could also be used to construct a reduced-order model of the numerical solution 35].

Sections 3.1 to 3.4 provide details on the calculation of the matrix $\mathbf{K}$.

\subsection{Spatial discretization}

The spatial derivatives in equation (2.1) are approximated using finite difference schemes:

$$
\frac{\partial \mathbf{q}}{\partial x}\left(x_{m}, x_{n}, t\right) \simeq \frac{1}{\Delta x} \sum_{j=-N}^{M} a_{j} \mathbf{q}_{m+j, n}(t), \quad \frac{\partial \mathbf{q}}{\partial y}\left(x_{m}, x_{n}, t\right) \simeq \frac{1}{\Delta y} \sum_{j=-N}^{M} a_{j} \mathbf{q}_{m, n+j}(t) .
$$

For a central scheme we have $M=N$. With solutions of the form $\mathbf{q} \sim \mathrm{e}^{-\mathrm{i} k x}$, the governing equations 2.9 and 2.10) are converted into a system of coupled ordinary differential equations:

$$
\frac{\partial \mathbf{r}}{\partial t}=\mathrm{i} \kappa \mathbf{G}_{x}\left(\mathbf{r}+\boldsymbol{\Sigma}_{y} \mathbf{g}\right)-\mathbf{G}_{y} \mathbf{D}_{y} \mathbf{r}-\boldsymbol{\Sigma}_{y} \mathbf{r}, \quad \text { and } \frac{\partial \mathbf{g}}{\partial t}=\mathbf{r},
$$

written for the vectors $\mathbf{r}$ and $\mathbf{g}$ which contain the values of $\mathbf{q}$ and $\mathbf{h}$ at the grid points. The matrices $\mathbf{G}_{x}$ and $\mathbf{G}_{y}$ are both block diagonal, with each block given by $\mathbf{F}_{x}$ and $\mathbf{F}_{y}$, respectively. The diagonal matrix $\boldsymbol{\Sigma}_{y}$ contains the values of the absorption coefficient $\sigma_{y}$. The matrix $\mathbf{D}_{y}$ represents the linear combinations introduced in (3.2) to calculate the derivative in the $y$ direction. The derivatives $\partial \mathbf{q} / \partial x$ and $\partial \mathbf{h} / \partial x$ in $(2.9)$ are approximated by $-\mathrm{i} \kappa(k) \mathbf{q}$ and $-\mathrm{i} \kappa(k) \mathbf{h}$ in the numerical model, where $\kappa$ is the effective wavenumber represented by the finite-difference scheme:

$$
\kappa(k)=\frac{\mathrm{i}}{\Delta x} \sum_{j=-N}^{N} a_{j} \mathrm{e}^{-\mathrm{i} k j \Delta x} .
$$

Note that in the absence of a PML we have $\sigma_{y} \equiv 0$ and equation (3.3) becomes

$$
\frac{\partial \mathbf{r}}{\partial t}=\left(\mathrm{i} \kappa \mathbf{G}_{x}-\mathbf{G}_{y} \mathbf{D}_{y}\right) \mathbf{r},
$$

which corresponds to the semi-discrete approximation of the standard conservation equations 2.1. 


\subsection{Impedance conditions}

The equations 2.4 and 2.5) for the auxiliary variables $\eta$ and $\xi$ are solved alongside equation (3.3). This can be written as an equation of the form

$$
\frac{\partial \mathbf{p}}{\partial t}=\mathbf{L}(k) \mathbf{p}(k, t)
$$

where the vector $\mathbf{p}$ gather all the unknowns of the problem: $\mathbf{r}, \mathbf{g}, \eta$ and $\xi$. The matrix $\mathbf{L}$ is constructed to combine equations (2.4), 2.5) and (3.3). In addition the rows of $\mathbf{L}$ corresponding to $\mathbf{q}_{0,0}$ are modified to represent either the direct implementation of the boundary condition (2.7), or the implementation based on characteristics $(2.8)$.

\subsection{Time integration}

We use a Runge-Kutta scheme with $P$ stages for the time integration with a time step $\Delta t$. It involves the following calculations:

$$
\begin{aligned}
\mathbf{p}_{0} & =\mathbf{p}(t), \\
\mathbf{p}_{l} & =\mathbf{p}(t)+\varphi_{l} \Delta t \mathbf{L}_{l-1}, \quad \text { with } l=1, \ldots, P, \\
\mathbf{p}(t+\Delta t) & =\mathbf{p}_{P},
\end{aligned}
$$

where the coefficient $\varphi_{l}$ characterise the integration scheme. Using equation 3.5 the solution $\mathbf{p}(t+\Delta t)$ at the next time step is obtained from the solution $\mathbf{p}(t)$ at the current time step as follows:

$$
\mathbf{p}(t+\Delta t)=\left[\mathbf{I}+\sum_{j=1}^{P} \gamma_{j}(\mathbf{L} \Delta t)^{j}\right] \mathbf{p}(t),
$$

where the coefficients $\gamma_{j}$ are given by $\gamma_{j}=\prod_{l=P-j+1}^{P} \varphi_{l}$ and are taken from reference [3].

\subsection{Selective filter}

Finally the last operation performed at each time step is the selective filtering used to damp poorly resolved waves. It is applied along each direction independently to obtain a filtered solution $\tilde{\mathbf{q}}$ :

$$
\tilde{\mathbf{q}}_{m, n}=\mathbf{q}_{m, n}-s \sum_{j=-N}^{N} f_{j} \mathbf{q}_{m+j, n}-s \sum_{j=-N}^{N} f_{j} \mathbf{q}_{m, n+j},
$$

where $s$ is the strength of the filter, and the coefficients $f_{j}$ of the stencils are taken from reference [2]. To account for this filtering, equation (3.6) is modified as follows:

$$
\mathbf{p}(t+\Delta t)=\left(\mathbf{I}-s \mu \mathbf{H}_{x}-s \mathbf{H}_{y}\right)\left[\mathbf{I}+\sum_{j=1}^{P} \gamma_{j}(\mathbf{L} \Delta t)^{j}\right] \mathbf{p}(t) .
$$

The matrix $\mathbf{H}_{x}$ is the identity matrix except for the two rows corresponding to $\eta$ and $\xi$ which are set to zero. $\mathbf{H}_{y}$ represents the linear combinations introduced by the filtering in the $y$ direction. The coefficient $\mu$ results from the filtering of the waveform $\mathrm{e}^{-\mathrm{i} k x}$ along the $x$ direction:

$$
\mu(k)=\sum_{j=-N}^{N} f_{j} \mathrm{e}^{-\mathrm{i} k j \Delta x} .
$$

Note that the filtering is only applied to the solution $\mathbf{q}$ and not to the auxiliary variables introduced by the liner model (the filtering of the variables $\eta$ and $\xi$ will be considered in section 5.1).

The matrix $\mathbf{K}$ introduced in equation (3.1) is readily identified as

$$
\mathbf{K}=\left(\mathbf{I}-s \mu \mathbf{H}_{x}-s \mathbf{H}_{y}\right)\left[\mathbf{I}+\sum_{j=1}^{P} \gamma_{j}(\mathbf{L} \Delta t)^{j}\right] .
$$




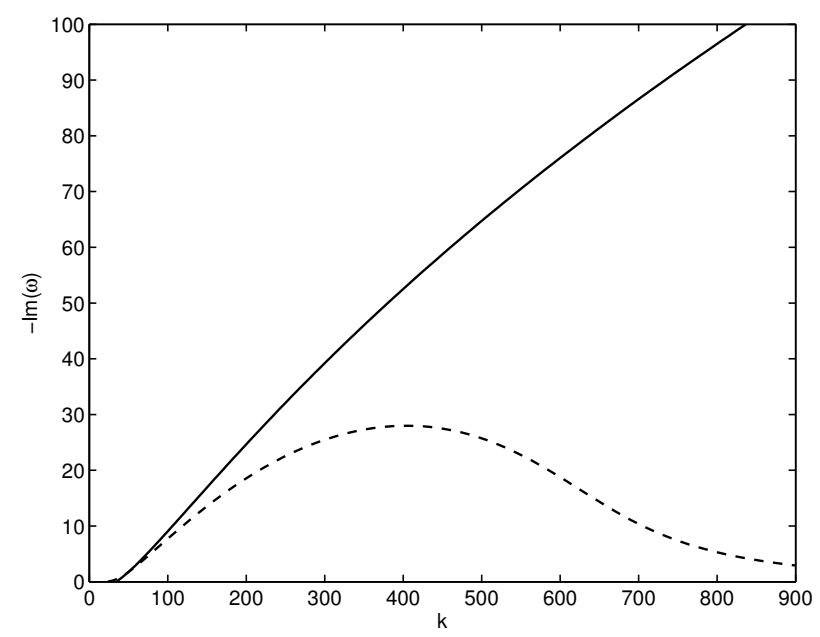

Figure 4.1: Exact temporal growth rate of the most unstable mode as a function of the wavenumber $k$ for the Myers condition (solid line) and for a real boundary layer with a linear profile and a thickness of $\delta=10^{-3}$ (dashed line).

\section{Properties of the instability}

We now use the dispersion analysis to study the properties of the instability observed in the numerical simulation. To this end the same test case as in section 2.5 will be used (results obtained with other parameters have been found to be consistent with those presented here).

Before presenting results for the numerical model it is useful to recall the properties of the instability in the continuous model. Figure 4.1 shows the growth rate $-\operatorname{Im}(\omega)$ of the most unstable mode, as well as the growth rate obtained for a boundary layer with a linear profile and a thickness $\delta=10^{-3}$ (these results are obtained using the methods presented in [5]). For the finite-thickness boundary layer this corresponds to a convective instability whose growth rate initially increases with $k$, reaches a maximum and then decay progressively. The Myers condition provides an approximation of the exact instability only for small wavenumbers $k$. More importantly, the growth rate increases indefinitely with $k$. The fact that the growth rate remains unbounded as $k$ increases is precisely what leads to the ill-posedness of the Myers condition [4]. This issue is due to the approximation of the real shear layer by a vortex sheet, and it has also been noted for open shear flows [14.

First, we show that the dispersion analysis of the numerical model introduced in section 3 is able to describe the instability observed in the time-domain simulation in figure 2.2 . For this purpose we compare the pressure distribution along the impedance surface $p(x, y=0, t)$ at two different time steps $t_{1}=400 \Delta t$ and $t_{2}=450 \Delta t$ (corresponding to figures $2.2 \mathrm{p}$ and $2.2 \mathrm{p}$ ). As can be seen in figure 4.2 , these take the form of a wavepacket with very short wavelength oscillations. The growth of this wavepacket between the two time steps is particularly rapid (almost two orders of magnitude).

We then calculate the spatial Fourier transforms $\hat{p}(k, y=0, t)$ of these solutions, as shown in figure 4.3 a. It is clear that these wavepackets are exclusively composed of wavenumber components between $k=200$ and $k=500$ approximately. The ratio between these two wavenumber spectra can be used to calculate the growth rate of the individual wavenumber components that compose the unstable wavepacket. This growth rate can then be compared against that predicted by the dispersion analysis. This comparison is shown in figure $4.3 \mathrm{~b}$ and demonstrate that the growth rate predicted by the dispersion analysis matches very well with what is observed in the numerical simulation.

It is also possible to use the complex-valued frequency $\omega(k)$ of the most unstable mode to construct the solution at $t_{2}$ from the solution at $t_{1}$. This is done by calculating the inverse Fourier transform of

$$
\hat{p}\left(k, y=0, t_{1}\right) \exp \left[i \omega(k)\left(t_{2}-t_{1}\right)\right],
$$

where the exponential accounts for the oscillation and growth of each wavenumber component $k$ between $t_{1}$ and $t_{2}$. The comparison between this solution and the actual pressure distribution observed in the numerical 
(a)

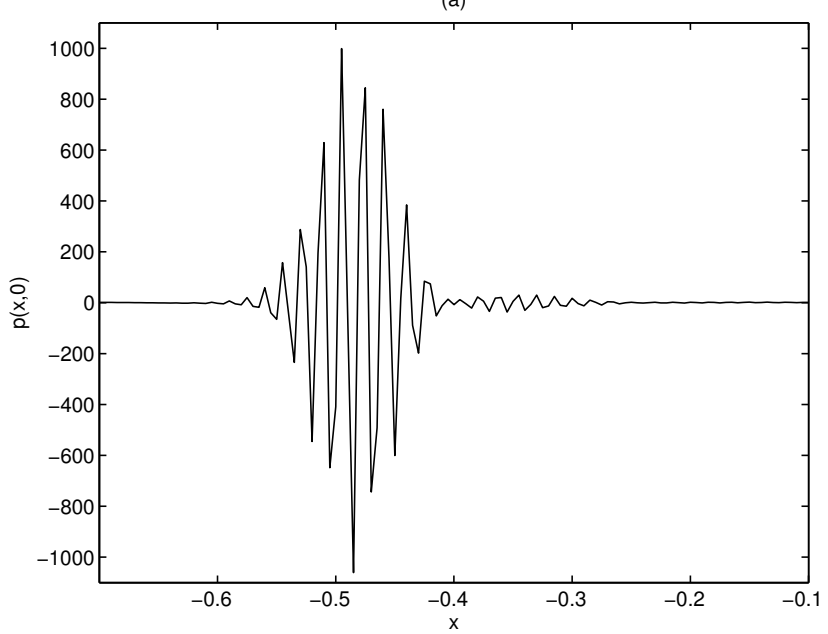

(b)

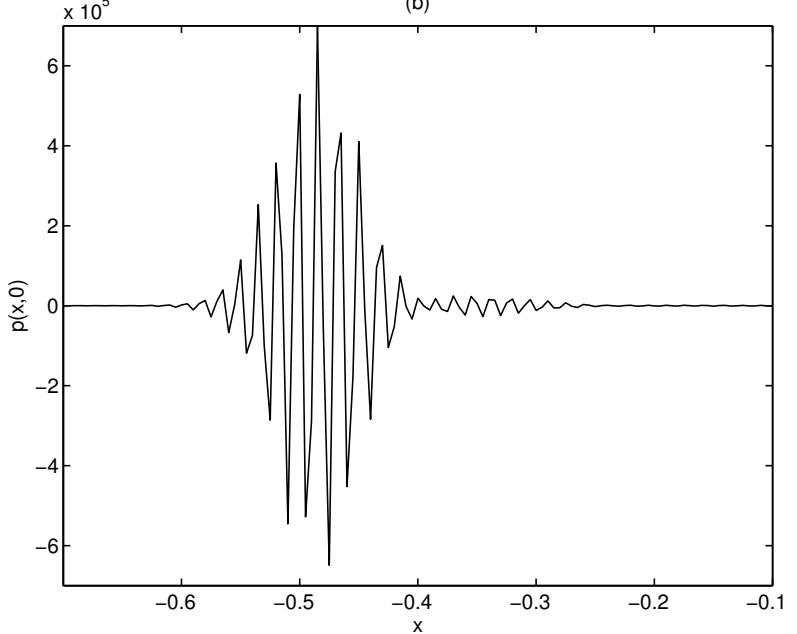

Figure 4.2: Instantaneous pressure $p(x, 0, t)$ along the impedance surface at (a) $t=400 \Delta t$ and (b) $t=450 \Delta t$. Note the rapid growth in amplitude between the two time steps.
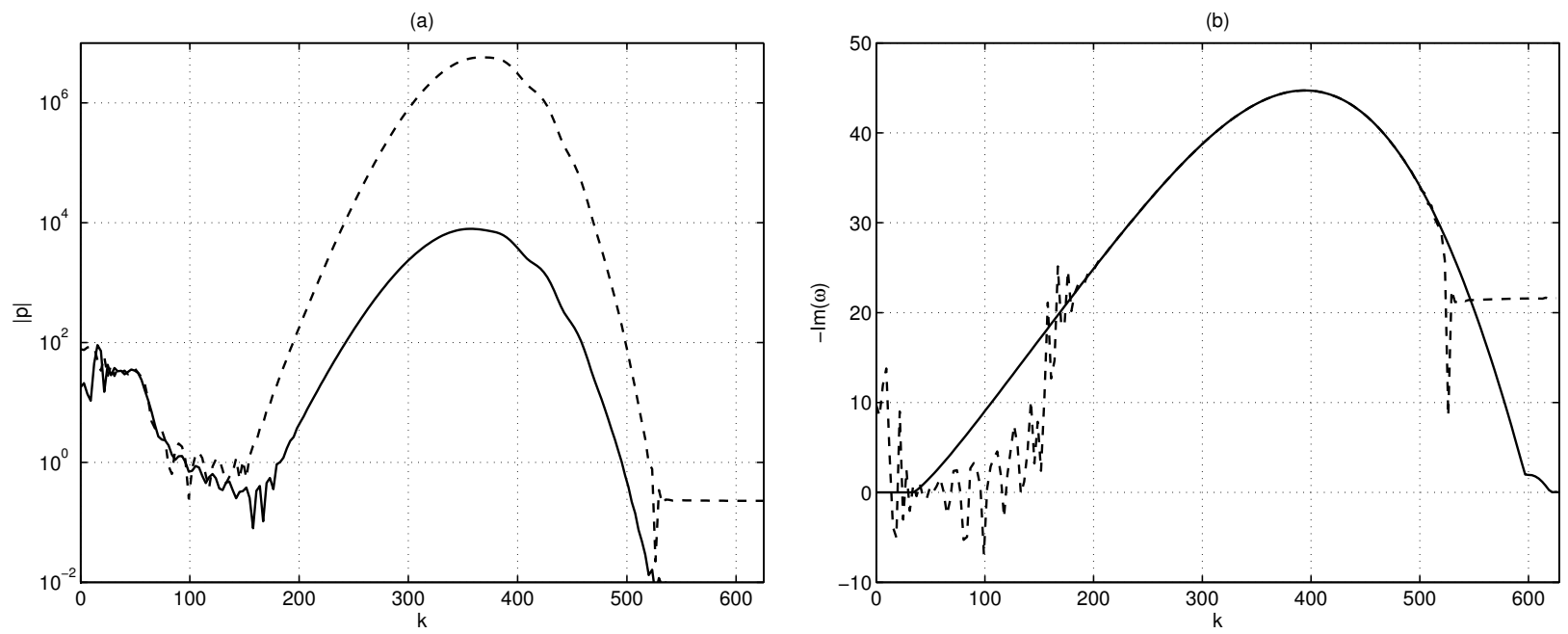

Figure 4.3: (a) Wavenumber spectrum of the instantaneous pressure $\hat{p}(k, 0, t)$ at $t=400 \Delta t$ (solid line) and $t=450 \Delta t$ (dashed line). (b) Growth rate of the wavenumber components predicted by the dispersion analysis (solid line) and calculated from the numerical simulation (dashed line). 

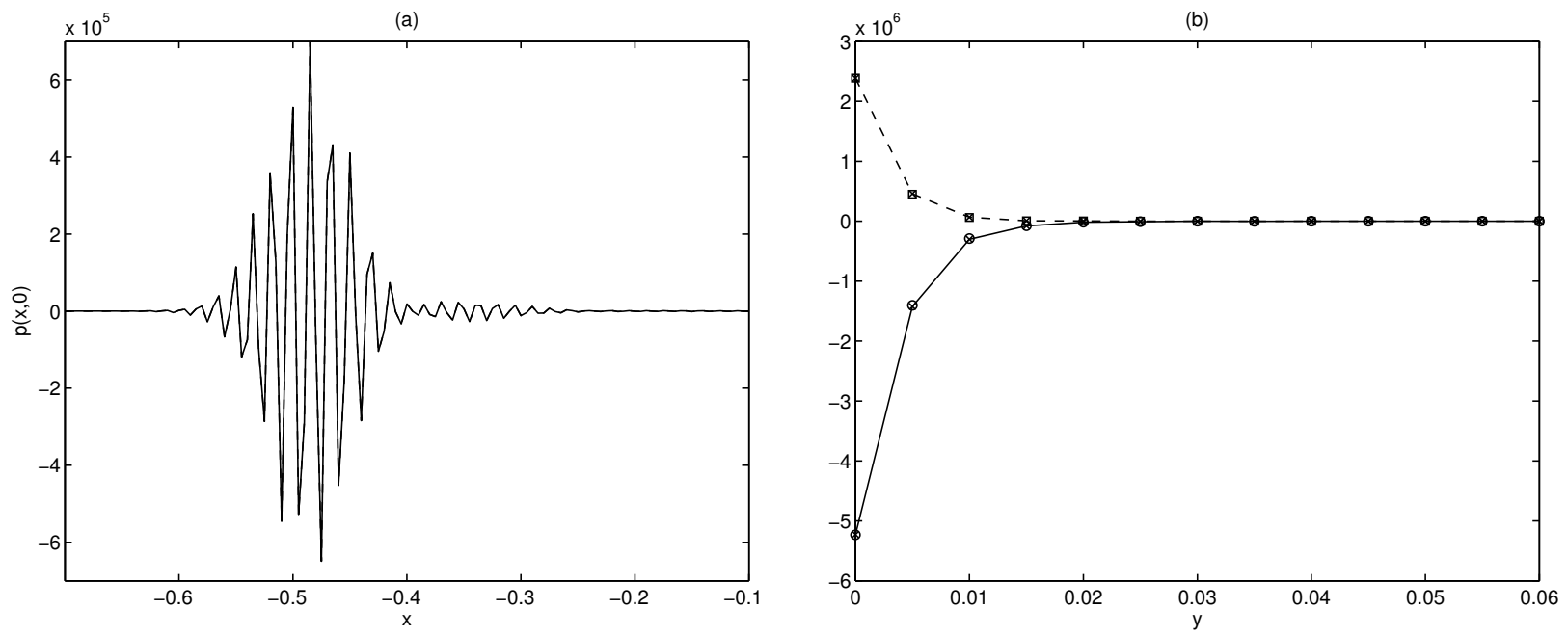

Figure 4.4: (a) Comparison between the pressure distribution along the impedance surface $p(x, 0, t)$ obtained from the simulation at $t=450 \Delta t$ (solid line) and constructed from the solution at $t=400 \Delta t$ (dashed line). (b) Comparison of the transverse pressure distribution $\hat{p}(k, y, t)$ associated with the strongest wavenumber component $k \simeq 356$. Numerical simulation: real part (०) and imaginary part $(\square)$; Dispersion analysis: real part (solid line) and imaginary part (dashed line).

simulation at $t_{2}$ is shown in figure 4.4 a, and it is clear that the two solutions match very well. Finally, we compare the transverse pressure distribution predicted by the dispersion analysis. This is shown in figure $4.4 \mathrm{~b}$ for the strongest wavenumber component $k \simeq 356$. The pressure distribution is found to match very well, up to a complex-valued constant, with the eigenvector associated with the strongest mode at $k \simeq 356$.

Now that we have shown that the dispersion analysis can predict very accurately the evolution of the instability observed in the numerical simulation, we discuss how the unstable mode in the numerical model relates to, and differ from, the instability found in the continuous model.

A fundamental difference between the numerical and continuous models is caused by the inherent limitations of any numerical approximation. Figure 4.5 a shows the effective wavenumber $\kappa$ as a function of $k$ for the finite-difference scheme used here. Due to the spatial sampling of the solution, we only have to consider the range $-\pi / \Delta x<k<\pi / \Delta x$. The key feature in figure $4.5 \mathrm{a}$ is that the effective wavenumber reaches a maximum value $\kappa_{\max }$ and then decays when the wave is not sufficiently resolved. So while the wavenumber $k$ can vary arbitrarily the numerical model will only include effective wavenumbers bounded by $-\kappa_{\max }$ and $\kappa_{\max }$. As a consequence the growth rate of the instability in the numerical model also remains bounded. Indeed, figure 4.5 shows that the growth rate first increases with $k$, but then reaches a maximum (corresponding to $\kappa_{\max }$ ) and then decay. Also shown in figure $4.5 \mathrm{p}$ is the case of a coarse grid where the growth rate is significantly reduced, since the corresponding $\kappa_{\max }$ is reduced when $\Delta x$ increases. This explains why the instability observed with the Myers condition tends to be weakened or removed when the grid resolution is reduced, as noted in the literature [21, 12].

In fact, the nature of the instability observed in the numerical model can be described, at least qualitatively, by the combination of the instability in the continuous model together with the effective wavenumber represented by the finite-difference scheme. This is shown in figure 4.5b by plotting $-\operatorname{Im}[\omega(\kappa(k))]$ where the dependence $\omega(\kappa)$ is taken from the continuous model and the effective wavenumber $\kappa(k)$ is given by (3.4). This estimate follows quite closely the actual growth rate observed in the full numerical model.

While the ill-posedness of the continuous model (due to the unbounded growth rate of the instability) is avoided in the numerical model thanks to the inability of the finite-difference approximation to represent the behaviour of large wavenumber components, this does not mean that the numerical model captures more accurately the instability of a real boundary layer. Figure 4.6 a shows the growth rate of the numerical model (either with a fine or a coarse grid) together with that of the continuous model with a finite-thickness boundary layer or with the Myers condition. It can be seen that the instability of the numerical model is 
(a)

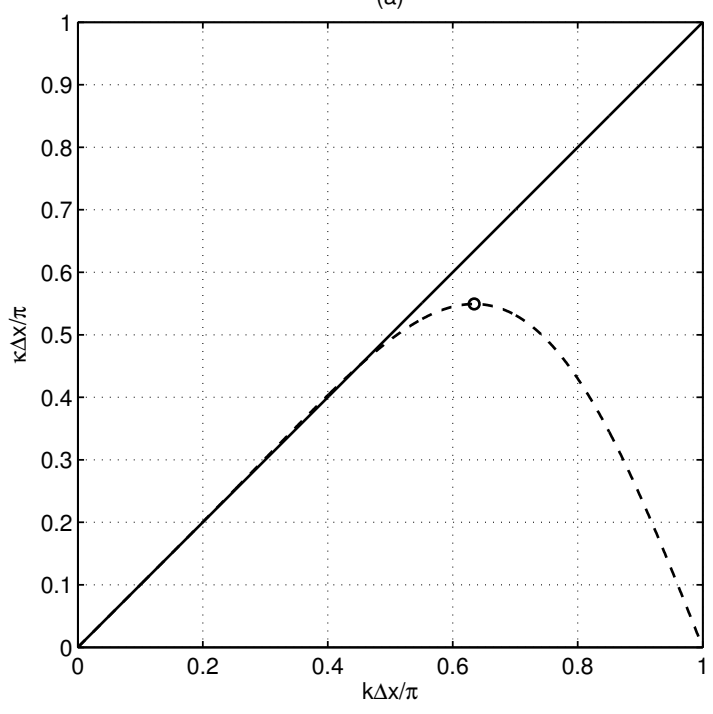

(b)

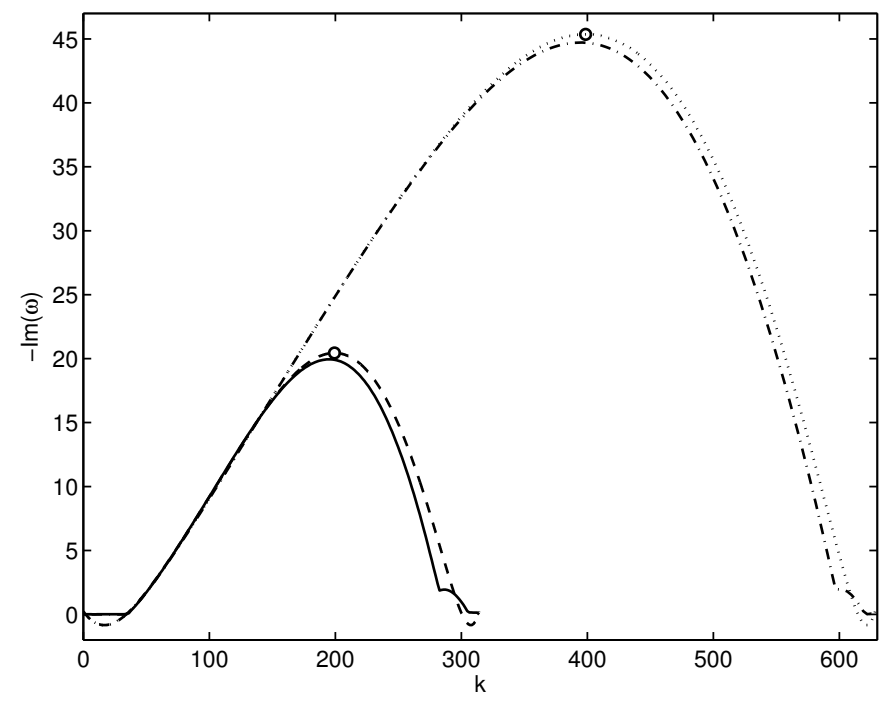

Figure 4.5: (a) Effective wavenumber $\kappa$ represented by the finite-difference approximation 3.4 as a function of the wavenumber $k$. (b) Growth rate of the most unstable mode with the coarse and fine grids (solid and dot-dashed lines, respectively), compared with the estimation - $\operatorname{Im}[\omega(\kappa(k))]$ for the coarse and fine grids (dashed and dotted lines, respectively) where $\omega(k)$ is given by the continuous model. The symbols $\circ$ show the location of $\kappa_{\text {max }}$.

very different from that of the real boundary layer. Figure 4.6 also illustrates that a fine grid leads to a stronger instability, since the exact solution is captured over a larger ranger of wavenumbers when $\Delta x$ is reduced.

In addition, the nature of the instability in the numerical model is fundamentally different from the continuous model in that the numerical model describes an absolute instability whereas the real boundary layer exhibits a convective instability. The group velocity of the instability is shown in figure 4.6b. For the numerical model with the Myers condition the real part of the group velocity vanishes for some real value of $k$. In fact it is possible to find a complex-valued wavenumber $\tilde{k}$ for which the group velocity of the unstable mode vanishes altogether. For the fine grid we have $\tilde{k} \simeq 394.5+0.4 \mathrm{i}$ and for the coarse grid $\tilde{k} \simeq 195.3+1.03 \mathrm{i}$. These particular wavenumber components correspond to absolute instabilities that will remain in the computational domain and render the solutions unusable. This is indeed the behaviour observed in figure 2.2. In contrast in figure $4.6 \mathrm{p}$ the group velocity of the instability wave associated with the real boundary layer remains positive, corresponding to a convective instability. For the continuous model with the Myers condition, the group velocity decreases as $k$ increases but remains positive.

\section{Properties of the numerical model}

The dispersion analysis can also be used to assess the impact of various aspects of the numerical schemes on the stability of the model.

\subsection{Use of filters and damping terms}

As reported in the literature [28, 12, 23, 30], it is quite common to use additional damping or filtering in the Myers condition to attempt to remove the instability associated with the liner in time-domain simulations (although the details are not always fully described). In principle the filter should not remove any physical instabilities. As shown above, the instability of the Myers condition has little resemblance with the real instability, so it could be justified to apply a strong filter to stabilise the numerical model. However, ultimately one would like to include a more accurate description of the real instability, either by using a modified impedance condition, or by resolving the boundary layer. 
(a)

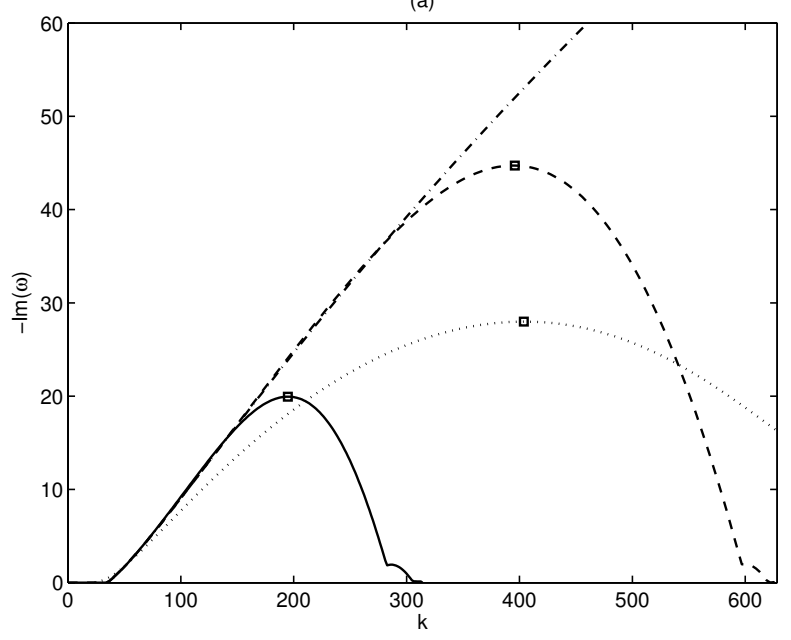

(b)

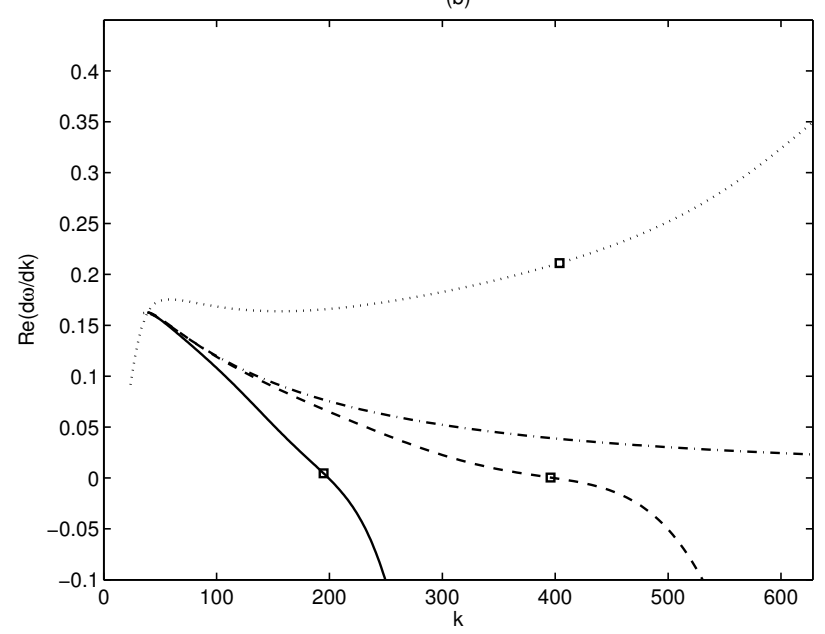

Figure 4.6: Growth rate (a) and group velocity (b) of the unstable mode as a function of the wavenumber $k$. Continuous model with the Myers condition (dot-dashed line) or a finite boundary layer with linear profile (dotted line). Numerical models with the Myers condition and a fine grid (dashed line) or a coarse grid (solid line). The symbols $\square$ show the most unstable wave components.

Ideally, the actual strength of the filter for the Myers condition should be adjusted on a case-by-case basis to ensure stability while minimising the impact on the acoustic part of the solution. However this can be quite difficult because the growth rate of the instability is very sensitive to the flow parameters, the properties of the impedance and the details of the numerical model. In practice the filter strength has generally been fixed to a large value with the expectation that it should be sufficient for most cases. There is the risk with this approach that the filtering of the Myers condition has an impact on the acoustic field, although some published results indicate that this is not necessarily the case [28, 12, 23, 30] and that accurate acoustic predictions can be obtained with a strong filter.

The dispersion analysis used here allows to measure the impact these techniques have on the stability of the numerical model. We consider a technique where a spatial filter is applied to the normal velocity calculated using (2.6) before it is used in equation (2.8). For the dispersion analysis, with solutions of the form $\mathrm{e}^{-\mathrm{i} k x}$, this amounts to multiply the last term in $(2.8)$ by a coefficient $1-\mu(k)$ with $\mu(k)$ given by (3.7). Figure 5.1 a shows $\mu(k)$ for the 7-point selective filter used here as well as for the low-order, 3-point filter used by Richter [29] (the coefficients are $f_{0}=1 / 2$ and $f_{1}=f_{-1}=-1 / 4$ ). Note that in [29] the 3-point filter was used to filter only a part of the normal velocity called the 'storage term'. Figure 5.1b shows the growth rate of the instability with and without these filters. The 7-point selective filter reduces somewhat the instability but it is the 3-point filter that is able to reduce significantly the instability (as can be seen in 5.1 this low-order filter has a strong impact even on well resolved wavenumber components). But, in this particular case, this filtering technique is not sufficient to remove completely the instability.

\subsection{Implementation of the boundary condition}

As explained in section 2.3.2 we considered a direct implementation (2.7) or an implementation where we modify the governing equations based on the characteristics (2.8). The latter was used for all the results presented so far. Figure 5.2 shows a comparison of the numerical modes obtained with these two approaches. The direct implementation leads to the presence of two very unstable modes (found for $\operatorname{Im}(\omega)<0)$ which can cause some difficulty during the simulation. But the use of the characteristics does not create such modes. It was indeed found that the direct implementation leads to the rapid growth of an instability in the time domain simulations. The importance of using the characteristics to formulate boundary conditions is well known, but this method does not seem to have been used systematically for the Myers condition in 
(a)

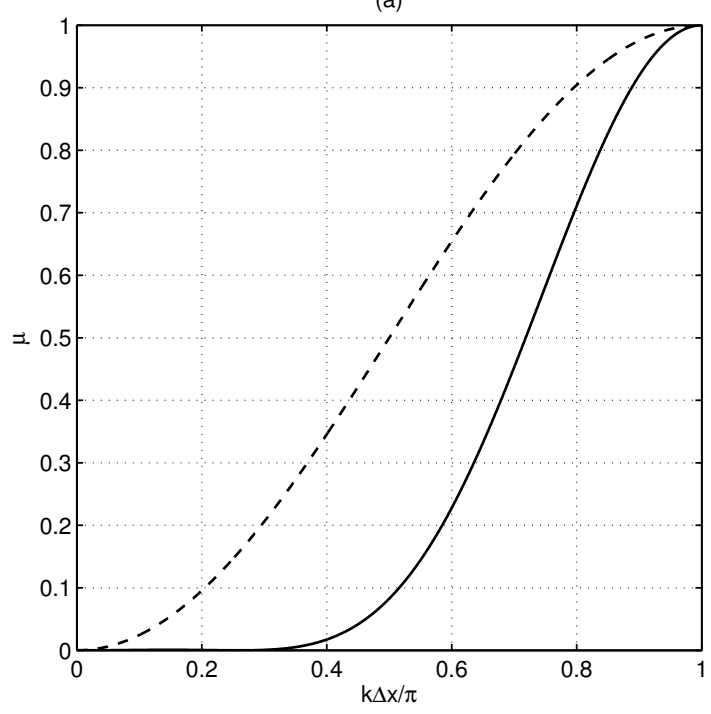

(b)

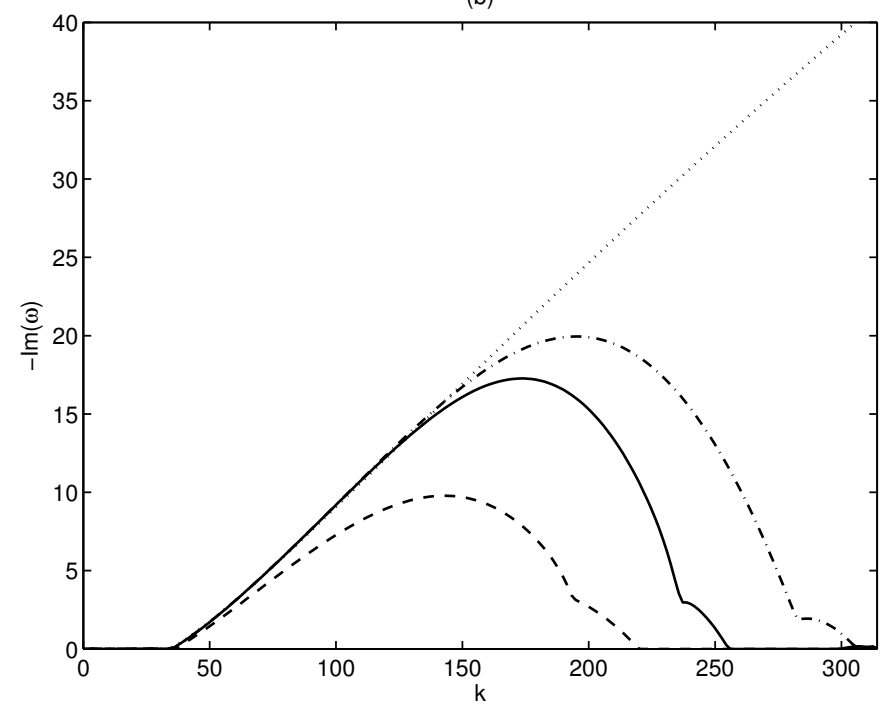

Figure 5.1: (a) Filter strength $\mu$ given by equation 3.7) as a function of the wavenumber $k$ for the 7-point selective filter (solid line) and for the 3-point filter (dashed line). (b) Growth rate of the unstable mode for the continuous model (dotted line), and for the numerical models with a 7-point filter for the normal velocity (solid line), the 3-point filter (dashed line), or no filtering (dot-dashed line).

time-domain simulations. So it is possible that some of the instabilities reported in the literature might be due not only to the Myers condition itself, but to the way this boundary condition has been implemented.

\subsection{Selective filter and time integration}

Another aspect that can have an impact on the stability of the numerical model is the use of a selective filter in the computational domain. There filters are designed to damp the spurious waves supported by high-order optimised schemes used in aeroacoustics. Since the most unstable mode in the numerical model corresponds to relatively poorly resolved wavenumbers, the selective filter is expected to have some effect on this instability. Figure 5.3 a shows the growth rate of the instability for different strengths of the selective filter. It can be seen that the growth rate can be slightly reduced by using a stronger filter. But to have more impact on the instability the strength of the selective filter would have to be increased to a point where even the well-resolved acoustic waves would be attenuated.

Finally we consider the effect of the time step on the instability. Figure $5.3 \mathrm{p}$ shows the growth rate of the instability for three different CFL numbers. There is no noticeable effect on the growth rate. This is because the real part of $\omega$ for the instability does not exceed 25, corresponding to approximately 62, 83 and 126 time steps per oscillation, indicating that the temporal evolution of the instability is very well resolved. While the instability in the numerical model is very strongly influenced by the spatial resolution (since it corresponds to relatively poorly resolved wavenumbers), it is well resolved in time and the time step has little influence on the properties of this instability (provided obviously that the CFL number remains acceptable).

As mentioned by one of the reviewers, in some cases the parameters of the liner model are such that a smaller time step is required than that indicated by the CFL number $\left(u_{0}+c_{0}\right) \Delta t / \Delta x$. For the massspring-damper model used here this would correspond to cases where the resonance frequency is high, thus necessitating a smaller time step. In the example shown here, the resonance frequency of the oscillator is close to 5, and so the dynamics of the liner model is also very well resolved in time. If this wasn't the case, one should expect the instability to also depend on the time step, and the numerical results to be inaccurate. 

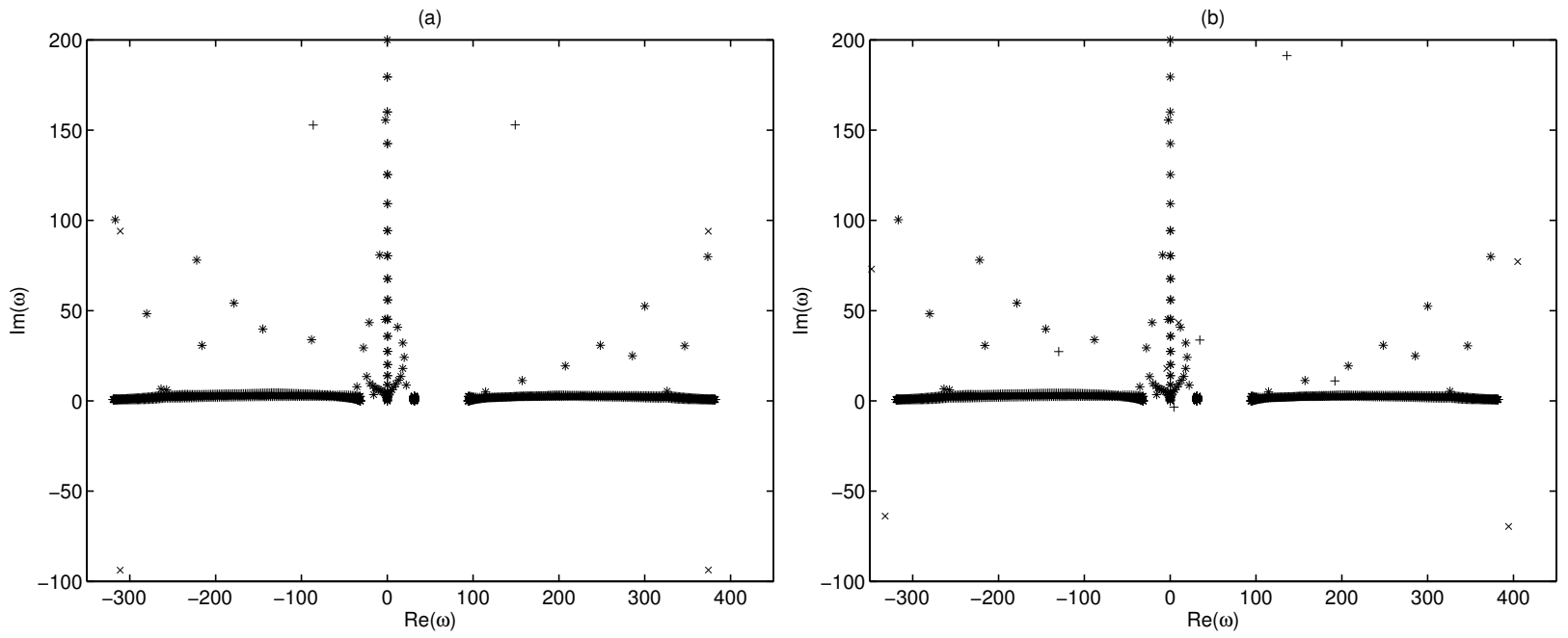

Figure 5.2: Angular frequency $\omega$ of the numerical modes for $k=20 \pi$ (corresponding to $k \Delta x=\pi / 10$ and 20 points per wavelength) and (a) a hard wall, or (b) a lined wall. $(\times)$ direct implementation 2.7 of the boundary condition, or $(+)$ characteristics implementation 2.8 .
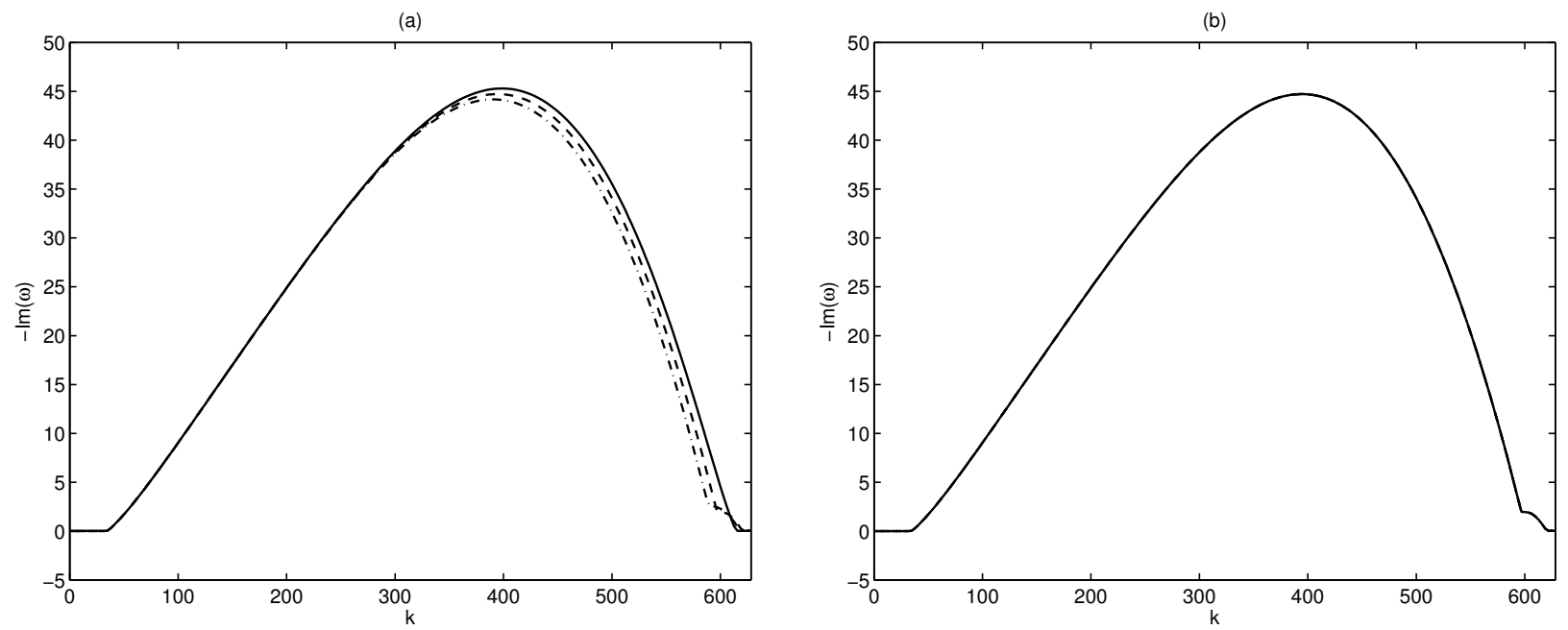

Figure 5.3: (a) Growth rate of the unstable mode with different strengths of the selective filter: $s=1.49 \times 10^{-2}$ (dot-dashed line), $s=7.47 \times 10^{-3}$ (dashed line), and no filtering $s=0$ (solid line). (b) Growth rate of the instability with different time steps based on a CFL number of 0.6 (solid line), 0.9 (dashed line) and 1.2 (dot-dashed line). 


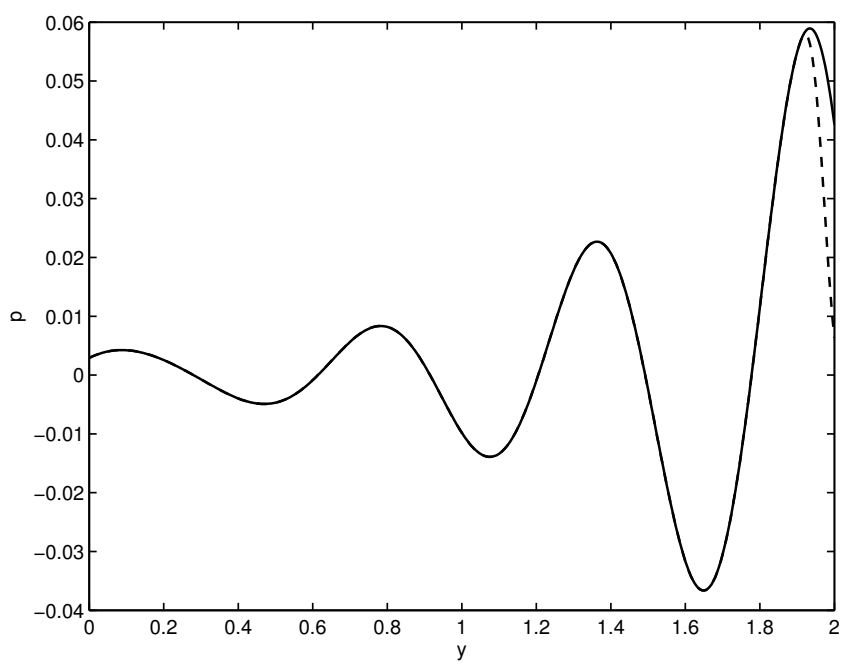

Figure 5.4: Pressure distribution $\hat{p}(k, y)$ associated with the numerical mode $\omega \simeq 46.92+0.57 \mathrm{i}$ for $k=30$ (dashed line) compared against the analytical solution (solid line).

\subsection{Acoustics modes of the numerical model}

The dispersion analysis is not limited to the study of unstable modes, but can also provide useful information about the accuracy with which the numerical model represents the acoustic part of the solution. For a given wavenumber $k$ there is a series of numerical modes corresponding to acoustic waves. In theory an acoustic plane wave in a uniform flow follows the dispersion relation

$$
\left(\omega-u_{0} k\right)^{2}=c_{0}^{2}\left(k^{2}+\gamma^{2}\right),
$$

where $\gamma$ is the wavenumber in the $y$ direction. With $k$ fixed, each mode of the numerical model corresponds to a specific value of $\omega$. Using (5.1) one can calculate the theoretical value of $\gamma$ corresponding to these values of $k$ and $\omega$, and the theoretical solution is of the form

$$
\hat{p}(k, y)=A \mathrm{e}^{-\mathrm{i} \gamma y}+B \mathrm{e}^{\mathrm{i} \gamma y}, \quad \text { with } \gamma=\sqrt{\left(\omega-u_{0} k\right)^{2} / c_{0}^{2}-k^{2}} .
$$

The coefficients $A$ and $B$ can be obtained by fitting this analytical expression to the numerical solution for $\hat{p}(k, y)$ which is part of the eigenvector associated with the mode under consideration. A least square method is used for this purpose.

To illustrate this procedure, we set $k=30$ and select a mode whose angular frequency is $\omega \simeq 46.92+0.57 \mathrm{i}$. Figure 5.4 shows a comparison between the theoretical expression $(5.2)$ and the actual pressure distribution found for this mode. Excellent agreement is observed, and the relative error between the numerical mode $\hat{p}(k, y)$ and the best fit based on $(5.2)$ is only $0.1 \%$. This shows that the numerical model captures very accurately the acoustic solution $(5.2)$ and the dispersion relation (5.1) used to define $\gamma$. Note that this result is obtained by excluding the grid points in the PML from the least square fit, since, by construction, the numerical solution in this region does not follow the theoretical dispersion relation (5.1). We can also calculate the corresponding reflection coefficient $R=A / B \simeq-0.445+0.186 \mathrm{i}$. The exact reflection coefficient is given by equation (3) in [17], and for this mode the relative error between the exact value and the numerical prediction is $0.7 \%$. This demonstrates that not only is the propagation of the acoustic waves above the liner well described, but the sound absorption by the liner, as predicted by the Myers condition, is also well captured in the numerical model.

This example illustrates how the dispersion analysis can be used to provide a quantitative assessment of the accuracy of the numerical schemes. 


\section{Conclusions}

In this paper, we investigate the numerical instability associated with the Myers boundary condition. A time-domain finite-difference model for the linearized Euler equations is used for this purpose, and applied to the simplistic case of a $2 \mathrm{D}$ point mass source (or, in other words, a 3D line source) in an otherwise uniform 2D flow over an acoustic lining. A detailed dispersion analysis of the numerical model is performed, incorporating all the aspects of the numerical model including the impedance boundary condition, the finitedifference approximations in time and space, the PML region and the selective filter. This dispersion analysis is used to study the properties of the instability and the results are compared with the dispersion analysis of the continuous model presented in reference [4]. The main findings are as follows:

- The unstable mode present in the numerical model is found to correspond to the instability associated with the Myers condition. However its properties differ significantly from that of the continuous model.

- Firstly, while the growth rate of the instability in the continuous model is unbounded (i.e. it increases indefinitely with the wavenumber $k$ ), it remains bounded in the numerical model. The effective wavenumber $\kappa(k)$ represented by any numerical approximation is bounded and only covers a range $-\kappa_{\max } \leqslant \kappa \leqslant \kappa_{\max }$. And since the growth rate of the theoretical instability increases monotonically with wavenumber, it follows that the growth rate in the numerical model is indeed bounded and the most unstable wavenumber component corresponds to $\kappa_{\max }$.

- As the grid is refined $\kappa_{\max }$ increases which leads to a larger growth rate for the instability in the numerical model. Therefore, this mechanism provides an explanation for the observations reported in the literature that the instability is more likely to appear with fine grids [21, 12].

- As a consequence, numerical models relying on the Myers condition in the time domain should not be expected to converge since any grid refinements will lead to a significant increase in the growth rate of the instability.

- We can also conclude that the inability of the finite-difference approximation to represent the behaviour of large wavenumber components is in fact the reason why the ill-posedness of the continuous model (due to the unbounded growth rate of the instability) is avoided in the numerical model.

- Another important difference with the continuous model is that the unstable mode in the numerical model corresponds to an absolute instability. Obviously this is particularly detrimental for practical simulations as this mode will remain in the computational domain and rapidly grow to cover the whole domain. This is also a significant difference with the behaviour of a real boundary layer which is convectively unstable.

- The dispersion analysis confirms that the use of a numerical filter for the Myers condition can have a significant impact on the growth rate of the instability, and can therefore be sufficient to render this mode stable in some cases. However, with a sufficiently fine grid it is possible that the growth rate of the instability will become too large to be fully cancelled by the numerical filtering. So the use of such filters is only viable when combined with the careful choice of a sufficiently coarse grid.

- The dispersion analysis shows that other numerical instabilities can also be present, for instance when using a direct implementation of the impedance condition. So it is possible that some of the instabilities reported in the literature might be due not only to the Myers condition itself, but to the way this boundary condition has been implemented. While the instability is strongly influenced by the spatial resolution, the time step has very little impact on its behaviour.

All the results presented here are for a uniformly lined surface. By comparing with an analytical solution (see figure 2.3 and by inspection of the numerical modes (in section 5.4) it was shown that the numerical model captures very accurately the reflection of acoustic waves as described by the Myers condition, and that it is the description of the instability, both in the continuous and the numerical models, that is the issue. 
It would be interesting to consider cases with a discontinuity of impedance, such as a transition between a lined and a hard wall, as it has been reported that such discontinuities can introduce additional forms of instabilities [29]. Crucially, liner discontinuities make it possible for a hydrodynamic instability that has developed over a liner to be scattered into acoustic waves by the edge of this liner.

From a practical point of view, filtering the instability over the liner can in some cases address the stability issues, provided the grid is not too refined. But this approach might not be valid when liner discontinuities are present, since the scattering of the instability by the liner edge would be missing and this would not lead to the correct, physical solution. For such situations it will be crucial to provide a more accurate description of the hydrodynamic waves above the liner. Fully resolving the boundary layer or using modified impedance conditions are two avenues that can be considered. Preliminary results on the use of the modified impedance condition proposed by Brambley [5] in time-domain simulations have recently been reported [9] and further progress will be presented in a subsequent paper.

Finally, it is hoped that the results presented in this paper demonstrate the benefits of conducting a dispersion analysis of numerical models with time-domain impedance conditions, in particular to assess the stability of the models. Similar analyses could be performed to assess and compare new methods or to revisit existing methods and better understand their properties, including impedance models [36, 28, 33, implementations of the boundary conditions [21, and numerical schemes (such as finite element, finite volume or spectral methods).

\section{Acknowledgements}

EJB gratefully acknowledges the support of the Royal Society for his University Research Fellowship.

A preliminary version of part of the results in this paper were presented as part of AIAA Paper 2013-2218 at the 19th AIAA/CEAS Aeroacoustics Conference in Berlin, Germany. 


\section{References}

[1] Y. Aurégan and M. Leroux. Experimental evidence of an instability over an impedance wall in a duct with flow. Journal of Sound and Vibration, 317:432-439, 2008.

[2] J. Berland, C. Bogey, O. Marsden, and C. Bailly. High-order, low dispersive and low dissipative explicit schemes for multiple-scale and boundary problems. Journal of Computational Physics, 224:637-662, 2007.

[3] C. Bogey and C. Bailly. A family of low dispersive and low dissipative explicit schemes for flow and noise computations. Journal of Computational Physics, 194:194-214, 2004.

[4] E.J. Brambley. Fundamental problems with the model of uniform flow over acoustic linings. Journal of Sound and Vibration, 322:1026-1037, 2009.

[5] E.J. Brambley. Well-posed boundary condition for acoustic liners in straight ducts with flow. AIAA Journal, 49(6):1272$1282,2011$.

[6] E.J. Brambley. Review of acoustic liner models with flow. In Société Française d'Acoustique, editor, Acoustics 2012 Nantes, pages 3429-3434, Nantes, France, 2012.

[7] E.J. Brambley. Surface modes in sheared boundary layers over impedance linings. Journal of Sound and Vibration, 332:3750-3767, 2013

[8] E.J. Brambley and G. Gabard. Reflection of an acoustic line source by an impedance surface with uniform flow. Journal of Sound and Vibration, 2013. Under review.

[9] E.J. Brambley and G. Gabard. Time domain simulations using the modified Myers boundary condition. In 19th AIAA/CEAS Aeroacoustics Conference, Berlin, Germany, 2013. AIAA paper 2013-2218.

[10] E.J. Brambley and N. Peake. Classification of aeroacoustically relevant surface modes in cylindrical lined ducts. Wave Motion, 43:301-310, 2006.

[11] M. Brandes and D. Ronneberger. Sound amplification in flow ducts lined with a periodic sequence of resonators. AIAA paper 95-126, 1995.

[12] N. Chevaugeon, J.-F. Remacle, and X. Gallez. Discontinuous Galerkin implementation of the extended Helmholtz resonator model in the time domain. In 12th AIAA/CEAS Aeroacoustics Conference, Cambridge, Massachusetts, USA, 2006. AIAA paper 2006-2569.

[13] C. Cossu and T. Loiseleux. On the convective and absolute nature of instabilities in finite difference numerical simulations of open flows. Journal of Computational Physics, 144:98-108, 1998.

[14] P.G. Drazin and W.H. Reid. Hydrodynamic stability. Cambridge University Press, 2004.

[15] W. Eversman. Approximation for thin boundary layers in the sheared flow duct transmission problem. Journal of the Acoustical Society of America, 53(5):1346-1350, 1973.

[16] W. Eversman and R.J. Beckemeyer. Transmission of sound in ducts with thin shear layers - convergence to the uniform flow case. Journal of the Acoustical Society of America, 52(1):216-220, 1972.

[17] G. Gabard. A comparison of impedance boundary conditions for flow acoustics. Journal of Sound and Vibration, 332:714$724,2013$.

[18] R.L. Higdon. Initial-boundary value problems for linear hyperbolic systems. SIAM Review, 28(2):177-217, 1986.

[19] F.Q. Hu. A stable, perfectly matched layer for linearized Euler equations in unsplit physical variables. Journal of Computational Physics, 173:455-480, 2001.

[20] K.U. Ingard. Influence of fluid motion past a plane boundary on sound reflection, absorption and transmission. Journal of the Acoustical Society of America, 31(7):1035-1036, 1959.

[21] H. Ju and K.-Y. Fung. Time-domain impedance boundary condition with mean flow effects. AIAA Journal, 39(9):1683$1690,2001$.

[22] J.W. Kim and D.J. Lee. Generalized characteristics boundary conditions for computational aeroacoustics. AIAA Journal, 38(11):2040-2049, 2000.

[23] X.D. Li, C. Richter, and F. Thiele. Time-domain impedance boundary conditions for surfaces with subsonic mean flows. Journal of the Acoustical Society of America, 119(5):2665-2676, 2006.

[24] D. Marx. A piecewise linear mean flow model for studying stability in a lined channel. Journal of Sound and Vibration, 331:3809-3823, 2012.

[25] D. Marx and Y. Aurégan. Effect of turbulent eddy viscosity on the unstable surface mode above an acoustic liner. Journal of Sound and Vibration, 332:3803-3820, 2013.

[26] D. Marx, Y. Aurégan, H. Bailliet, and J.-C. Valière. PIV and LDV evidence of hydrodynamic instability over a liner in a duct with flow. Journal of Sound and Vibration, 329:3798-3812, 2010.

[27] M.K. Myers. On the acoustic boundary condition in the presence of flow. Journal of Sound and Vibration, 71(3):429-434, 1980.

[28] Y. Özyörük, L. Long, and M.G. Jones. Time-domain numerical simulation of a flow-impedance tube. Journal of Computational Physics, 146:29-57, 1998.

[29] C. Richter. Liner impedance modelling in the time domain with flow. Ph.D. thesis, Technical University of Berlin, Germany, 2009.

[30] C. Richter, F. Thiele, X. Li, and M. Zhuang. Comparison of time-domain impedance boundary conditions for lined duct flows. AIAA Journal, 45(6):1333-1345, 2007.

[31] C. Richter and F.H. Thiele. The stability of time explicit impedance condition. In 13th AIAA/CEAS Aeroacoustics Conference, Rome, Italy, 2007. AIAA paper 2007-3538.

[32] S.W. Rienstra. A classification of duct modes based on surface waves. Wave Motion, 37:119-135, 2003. 
[33] S.W. Rienstra. Impedance models in time domain, including the extended Helmholtz resonator model. In 12th AIAA/CEAS Aeroacoustics Conference, Cambridge, MA, USA, 2006. AIAA paper 2006-2686.

[34] S.W. Rienstra and M. Darau. Boundary-layer thickness effects of the hydrodynamic instability along an impedance wall. Journal of Fluid Mechanics, 671:559-573, 2011

[35] G. Serre, P. Lafon, X. Gloerfelt, and C. Bailly. Reliable reduced-order models for time-dependent linearized euler equations. Journal of Computational Physics, 231:5176-5194, 2012.

[36] C.K.W. Tam and L. Auriault. Time-domain impedance boundary conditions for computational aeroacoustics. AIAA Journal, 34(5):917-923, 1996.

[37] C.K.W. Tam, H. Ju, and E.W. Chien. Scattering of acoustic duct modes by axial liner splices. Journal of Sound and Vibration, 310:1014-1035, 2008.

[38] C.K.W. Tam and J.C. Webb. Dispersion-relation-preserving finite difference schemes for computational acoustics. Journal of Computational Physics, 107:262-281, 1993.

[39] B.J. Tester. Some aspects of "sound" attenuation in lined ducts containing inviscid mean flows with boundary layers. Journal of Sound and Vibration, 28(2):217-245, 1973.

[40] L.N. Trefethen. Group velocity in finite difference schemes. SIAM Review, 24(2):113-136, 1982.

[41] R. Vichnevetsky and J.B. Bowles. Fourier analysis of numerical approximations of hyperbolic equations. Society for Industrial and Applied Mathematics, 1987. 